\title{
A Large TPC Prototype for an ILC Detector
}

\author{
Klaus DEHMELT ${ }^{* \dagger}$ \\ DESY \\ E-mail: klaus.dehmelt@desy.de
}

A Time Projection Chamber (TPC) is a candidate for the central tracker of the future International Linear Collider (ILC) detectors. TPCs have already demonstrated very good performance in past collider experiments. However, the tracking system of the ILC should have a very good track momentum resolution $\left(\Delta\left(1 / p_{t}\right) \sim 5 \times 10^{-5} \mathrm{GeV} / \mathrm{c}\right)$, which is an order of magnitude more precise than in previous experiments. To achieve this resolution, the Linear Collider TPC (LCTPC) groups are pursuing R\&D activities to determine the best state-of-the-art technology for the TPC using Micro Pattern Gas Detectors (MPGD) readout instead of the Multiwire Proportional Chamber (MWPC) readout. The MPGDs under investigation are the Gas Electron Multiplier (GEM) and the Micromesh Gaseous (MICROMEGAS) detectors as well as a new concept combining a gas amplification on top of a CMOS pixel readout chip (TimePix). To study these technologies, a Large Prototype TPC (LPTPC) has been built, with a diameter of about $750 \mathrm{~mm}$ and a length of about $600 \mathrm{~mm}$, which allows to measure tracks with up to 125 space points with pad readout. Since end of 2008, the LPTPC has been inserted into a 1.25 Tesla superconducting magnet, installed in a DESY test beam area. The LPTPC, alternatively equipped with the GEM or the MICROMEGAS readout, is exposed to an electron beam of up to $6 \mathrm{GeV}$. With both technologies the preliminary results look very promising. A first TimePix endplate module, consisting of 8 chips and a triple-GEM stack has been tested in June 2009 at the LPTPC. The LPTPC is not only a testing bed for several readout techniques based on MPGDs it is also an opportunity to understand the issues which arise when constructing such a large TPC. We will report on the setup, the production and the commissioning of the LPTPC as well as the first results of the test beams with the different readout technologies.

European Physical Society Europhysics Conference on High Energy Physics July 16-22, 2009

Krakow, Poland

\footnotetext{
* Speaker.

$\dagger$ On behalf of the LCTPC collaboration.
} 


\section{Introduction}

A detector at the International Linear Collider (ILC) will have a high-precision tracking system inside a calorimeter system, and both systems will have very high granularity. These will be contained in the detector solenoid which will produce the high magnetic fi eld (4T) needed to reduce backgrounds at the vertex and to enable very good momentum resolution. TPCs based on Micro Pattern Gas Detectors (MPGD) are described in the ILC Reference Design Report [2] and have dimensions of $2.8-4 \mathrm{~m}$ in diameter and $3-4.6 \mathrm{~m}$ in length. They are to provide 200 space points with pad readout, along a particle track with the $\mathrm{R} \phi$ spatial resolution of $100 \mu \mathrm{m}$ per row or better. The momentum resolution of $\delta(1 / p t) \leq 5 \times 10^{-5}(\mathrm{GeV} / \mathrm{c})^{-1}$ is envisaged in the magnetic fi eld of 3-4 T.

\section{The Large TPC Prototype}

Several relevant topics towards the ILC detector have been studied at small and medium sized TPC prototypes and are now being pursued with a Large Prototype (LP) of a TPC. The LP has a diameter of about $750 \mathrm{~mm}$ and a length of about $600 \mathrm{~mm}$. This prototype fi ts into a superconducting magnet (permanent current magnet, PCMAG) that has been installed in a test beam area at DESY in Hamburg. PCMAG delivers a magnetic fi eld up to $1.25 \mathrm{~T}$. The test beam consists of electrons with a momentum of up to $6 \mathrm{GeV} / \mathrm{c}$ and allows to measure tracks with the LP of up to 125 space points with pad readout.

\subsection{The Field Cage}

Part of the LP is a fi eld cage (FC), which is made out of composite materials [6]. The materials were chosen such that they guarantee a maximum of stability, though providing a minimum of material for the traversing particles. The homogeneous electrical drift for the ion and electron clouds in the TPC volume is provided through a series of fi eld strips, which have to be arranged such that the relative distortions of the fi eld are below $10^{-4}$ within the drift volume. This can be achieved with mirror strips that lie on an intermediate potential.

\subsection{Anode Endplate}

Endplates were designed such that amplifi cation modules can be mounted in a pattern that is a circular subsection of a possible TPC for the ILC [ 4 ]. The endplates allow to position the modules to an accuracy of better than $50 \mu \mathrm{m}$. Several areas have been cut out in order to implement further devices for usage with the TPC, e.g. laser insertion holes.

\subsection{Amplification Modules}

To realize the excellent space point resolution, a TPC with MPGD readout instead of the Multiwire Proportional Chamber (MWPC) readout is needed. The MPGD under consideration are Gas Electron Multiplier (GEM) [5] and Micromesh Gas detector (Micromegas) [6] with standard signal pads as well as with CMOS pixel (TimePix) readout [7]. For the Micromegas option the resistive bulk technology has been used [8]. Modules will also be equipped with gating devices in order to reduce the ion back flow into the sensitive volume. 


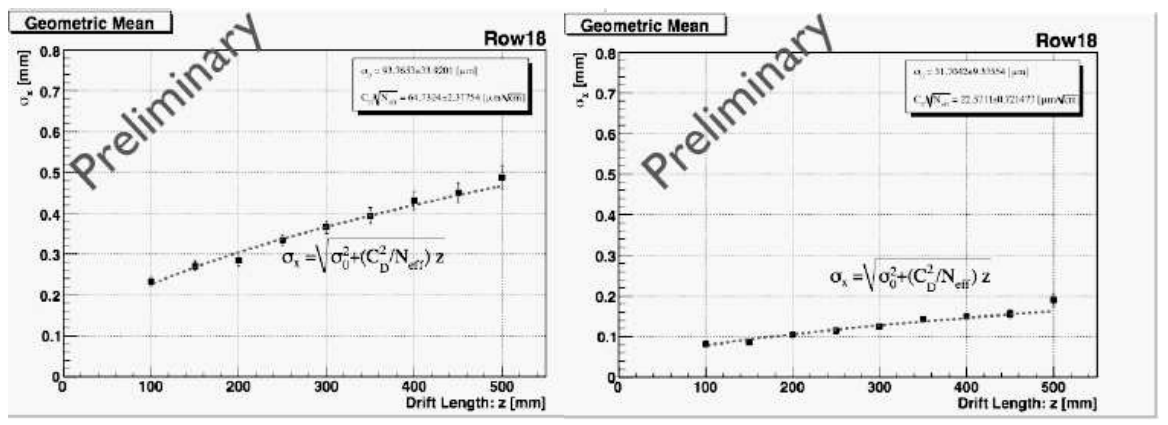

Figure 1: Single point resolution with $B=0 \mathrm{~T}$ (left) and with $\mathrm{B}=1 \mathrm{~T}$ (right) for a double GEM structure.

\subsection{Test Procedures}

Correction techniques for E- and B-fi eld distortions have to be evaluated and the LP in connection with PCMAG offer a good opportunity to test these techniques since PCMAG does not have a return yoke, thus providing a signifi cant fi eld inhomogeneity. A system using photoelectrons produced at the cathode can be used for verifying and correcting these distortions. Gas mixtures are being tested according to their performance, e.g. low diffusion, suffi cient number of primary electrons and small electron attachment, suffi cient large drift velocities of electrons and ions in acceptable fi elds and to test gating conditions in the case of GEM gating.

\subsection{Readout Electronics}

The LP is operating with a large number of channels that read out the signals on either pads or CMOS pixels. For the GEM option this readout system is based on readout electronics that was developed for the ALICE experiment at the LHC, ALICE TPC Read Out (ALTRO) [9]. Starting with 200 ALTRO chips, which corresponds to 3200 channels, the chip is digitizing the TPC signals with a sampling frequency of $40 \mathrm{MHz}$. The readout system will be extended by 1600 chips with $25 \mathrm{MHz}$ sampling rate. In order to adopt this chip to the specifi cs of a MPGD based TPC, a new charge sensitive preamplifi er has been developed (PCA16). Furthermore, a TPC readout electronics will be tested, where the time of arrival and charge of the signals on the pads are measured with a TDC [10]. The charge is measured indirectly, by means of a charge-to-time converter. For the Micromegas option the AFTER-based TPC electronics is being used, from T2K, which has been successfully commissioned. The number of channels is 1728 per module, using an average pad size of $3.2 \times 7 \mathrm{~mm}^{2}$

\section{Summary}

A large prototype of a TPC has been constructed and is being tested and commissioned. The LP is a testing bed for several readout techniques based on MPGD in connection with a test of mechanical feasibility of a large TPC. A sophisticated ensemble has been established in order to perform R\&D whose goal is to obtain confi dence that a TPC with MPGD readout will be a suitable central tracking device for an ILC detector. The LP has been exposed to electrons emerging from the DESY accelerator, both with modules based on Micromegas as well as GEM amplification 


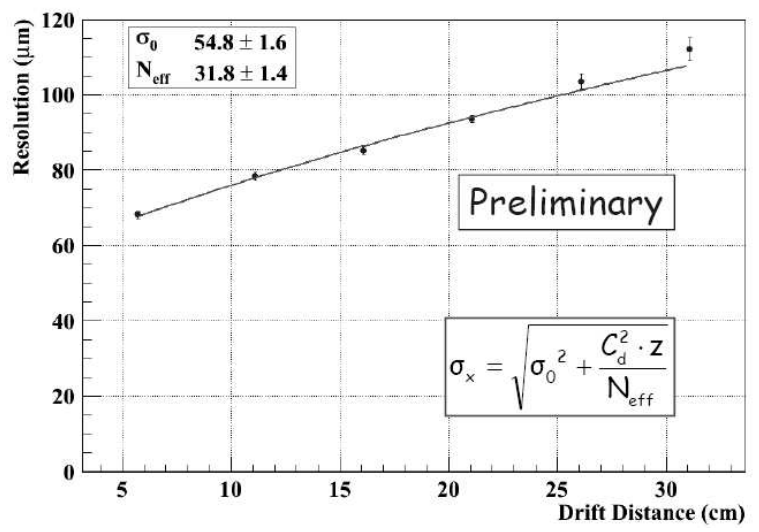

Figure 2: Single point resolution with $\mathrm{B}=1 \mathrm{~T}$ for a Micromegas structure.

structures. First results of these tests (Fig. 1, 2) are promising and providing a good basis for further activities.

\section{References}

[1] TPC R\&D for an ILC Detector (Status Report for the WWSOC R\&D Tracking Review, Beijing, 6-7 February 2007) LC-DET-2007-005, http://www-flc.desy.de/lcnotes

[2] ILC Reference Design Report, ILC-Report-2007-001, http://www.linearcollider.org

[3] T.Behnke et al., JRA2 Milestone: Field Cage Available, Eudet-Memo-2008-41, http://www.eudet.org/e26/e28/e615/e832/eudet-memo-2008-41.pdf

[4] D. Peterson, http://www.lepp.cornell.edu/d̃pp/linear_collider/LargePrototype.html

[5] F. Sauli, GEM: A New Concept for Electron Amplification in Gas Detectors,Nucl.Instrum.Meth. A386(1997)531.

[6] Y.Giomataris et al.,Micromegas: A High Granularity Position Sensitive Gaseous Detector for High Particle Flux Environments, Nucl.Instrum.Meth. A376(1996)29.

[7] M. Campbell et al., Detection of single electrons by means of a Micromegas-covered MediPix2 pixel CMOS readout circuit, Nucl.Instrum.Meth. A540(2005)295.

[8] M. Dixit et al., Nucl.Instrum.Meth. A518(2004)721.

[9] ALICE Collaboration, A Large Ion Collider Experiment, ALICE TPC-Design Report, ISBN 92-9083-155-3, December 1999, CERN, Geneva, Switzerland. http://aliceinfo.cern.ch/

[10] A. Kaukher, Ph.D. thesis, University of Rostock. 